\title{
OSTEOSYNTHESIS MATERIALS IN MAXILLOFACIAL SURGERY: REJECTION, REMOVAL, CORROSION AND PARTICLE DETECTION RATES
}

\author{
Samy El Bachaoui ${ }^{1 a} \mathbb{( D}$, Constantinus Politis ${ }^{1 b^{*}}$ (1) \\ 'Department of Oral and Maxillofacial Surgery, Faculty of Medicine, University of Leuven, Leuven, Belgium \\ aMD, Master's Student in Medicine; e-mail: samy.elbachaoui@gmail.com; ORCIDiD: https://orcidorg/0000-0003-4492-7564 \\ bMD, DDS, MM, MHA, PhD, Professor \& Chairperson; e-mail: constantinus.politis@uzleuven'be; ORCIDiD: https://orcidorg/0000-0003-4772-9897
}

ABSTRACT

di) https://doi.org/10.25241/stomaeduj.2020.8(2).art.4

Background Titanium is traditionally the material of choice for osteosynthesis in maxillofacial surgery and has a wide array of application in this field. Conversely, a growing interest for alternative fixation methods has emerged in the literature. Promising results have been reported for 3D-designed and manufactured (CAD/CAM) titanium materials, whereas the use of biodegradable materials seems to be a more controversial topic.

Objective To conduct a narrative review on the complications related to osteosynthesis materials in maxillofacial surgery in terms of rejection-, removal-, corrosion- and particle detection rates.

Data Sources A literature search was performed in April 2020 using the electronic database PubMed (National Library of Medicine, NCBI). The search included studies published between 1999 and March 2019. Study Selection Articles were eligible for inclusion when data for the outcomes of interest were available.

Data Extraction Complication rates including rejection-, removal-, corrosion- and particle detection rates were extracted.

Data Synthesis The data were synthesized and analyzed according to the different types of osteosynthesis materials and fixation methods. Finally, the results were summarized and recommendations were listed for different types of surgical indications.

\section{KEYWORDS}

Mandibular Reconstruction; Bone Plates; Postoperative Complications; Surgical Wound Dehiscence; Corrosion.

\section{INTRODUCTION}

The use of plates and screws for osteosynthesis is the golden standard in maxillofacial surgery. In the literature, a wide range of different types of materials are used for different purposes, and each type of material has its own properties. Stainless steel was the first type of material to dominate the market, but it has been left behind due to its toxic and corrosive properties [1]. Stainless steel was replaced by titanium as the golden standard for osteosynthesis, which was found to be much more efficient, because of its non-toxicity and corrosion-resistance [1]. Since the introduction of titanium, an important evolution has been witnessed from standard titanium plates and screws to 3D -designed and -manufactured (CAD/CAM) titanium plates and screws $[2,3]$. Other contenders in this field are the bioresorbable materials, which in theory are interesting because of their bioresorbable aspects and the possibility to avoid a second surgery to remove the osteosynthesis material [4,5]. Several studies have analyzed these different types of materials in detail, proving each material has its own advantages and disadvantages. However, an overview comparing the complication rates of the different material types is lacking. The aim of this narrative review was to compare the complication rates related to osteosynthesis materials and reconstruction plates in terms of rejection, removal, corrosion and particle detection in soft tissues.

\section{MATERIALS AND METHODS}

PubMed was searched for articles that compared the different outcomes of interest from human results concerning osteosynthesis material and reconstruction plates. A lot of different outcomes are described in the literature, and not all authors use the same terminology. The term 'rejection' could not be found as such in the database, therefore it was redefined as a collective term for different more 


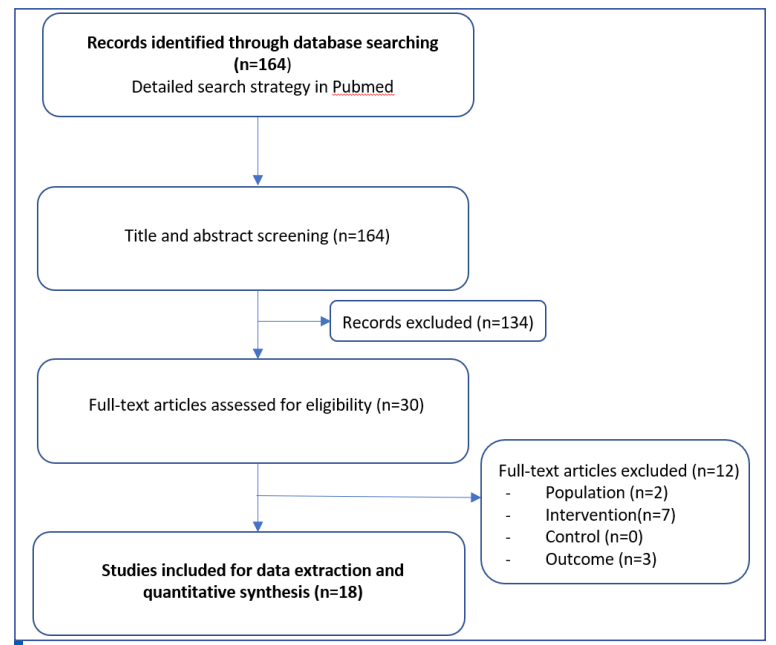

Figure 1. Flowchart of the study selection process.

commonly used outcomes in the literature such as 'infection', 'wound dehiscence', 'plate exposure', or 'screw loosening'. The search was split into 2 parts: the outcomes of 'rejection' and 'removal' were searched simultaneously (search A), as well as the outcomes of 'corrosion' and 'particle detection' (search B). The main keywords used to build the search strategy were: "rejection", "equipment failure", "infection", "screw loosening", "plate extrusion", "plate exposure", "wound dehiscence", "device removal", "plate removal" and "hardware removal" for search A; "corrosion", "biocompatible materials/chemistry", "titanium/chemistry", "particle detection", and "pigment deposition" for search B (see Appendix I for full search strategy). An initial review was done based on title and abstract with a restriction in time of 10 years for search $A$ and no restriction in time for search B. Potential articles were then examined in full text. A total of 15 studies were included in search $A$, and three studies in search B (Fig. 1). Articles were eligible for inclusion when data for the outcomes of interest were available. Further restrictions for the articles are listed in the exclusion criteria.

\subsection{Selection Criteria}

2.1.1. Inclusion Criteria

Search A:

- Population: $\mathrm{N}>100$

- Intervention: the use of osteosynthesis material/ reconstruction plates for any type of maxillofacial procedure

- Outcome: infection, screw loosening, wound dehiscence, removal of osteosynthesis material/ reconstruction plates

- 10-year time restriction (2010-2020)

- Study type: systematic reviews (and metaanalysis), RCT, experimental controlled studies, observational studies.

O Search B:

- Intervention: the use of osteosynthesis material/ reconstruction plates for any type of maxillofacial procedure

- Outcome: corrosion, particle detection in lymph nodes/soft tissues

- Study type: systematic reviews (and metaanalysis), RCT, experimental controlled studies, observational studies.

\subsubsection{Exclusion Criteria}

O Search A:

- Population: $\mathrm{N}<100$

- Publication dates older than the past 10 years

- Study type: case reports, expert opinions, animal studies, ex vivo experiments

O Search B:

- Study type: case reports, expert opinions, animal studies, ex vivo experiments

\section{RESULTS}

\subsection{Rejection- and Removal Rates}

3.1.1. Titanium Materials

3.1.1.1. Miniplates vs Reconstruction Plates

2 studies were found that compared these fixation systems. One systematic review included 5 studies with 511 cases who underwent vascularized osteocutaneous flap reconstruction of the mandible [6]. Patients with miniplates had a slightly higher rate of complications than did cases using reconstruction plates $(R R=1.1)$, but no significant difference in complication rates was found between the groups. The second retrospective study included 682 patients with fractures of the mandibular symphysis/body [7]. Both plating techniques used in this study (1 large plate vs 2 miniplates) show very good outcomes, but the application of a second bone plate increased the incidence of wound dehiscence, plate exposure, and need for plate removal significantly. Overall, a higher rate of complications was found in the miniplate groups. Therefore, one large reconstruction plate is recommended above titanium miniplates as a treatment for vascularized osteocutaneous flap reconstruction of the mandible and for fractures of the mandibular symphysis/body.

3.1.1.2. Single Miniplate vs Double Miniplate

2 studies compared the use of a single- vs a double miniplate system in the treatment of a mandibular angle fracture. Both studies concluded that a single miniplate fixation system resulted in good stability and fewer postoperative complications, including wound problems/dehiscence, infection, screw loosening, plate fracture and hardware removal $[8,9]$. 3.1.1.3. $2.0 \mathrm{~mm}$ Locking Reconstruction Plates

2 studies were included that used $2.0 \mathrm{~mm}$ locking reconstruction plates for mandibular reconstruction. The first study included 307 patients who had undergone osteocutaneous free flap reconstruction with $2.0 \mathrm{~mm}$ locking plate fixation following mandibular resection for benign or malignant neoplasia or osteoradionecrosis [10]. Plate removal was necessary in $27 \%$, most likely due to surgical site infection or fistula formation. The second study included 162 patients with segmental resections of the mandible reconstructed with angular stable plates [11]. They compared $2.0 \mathrm{~mm}$ with 2.5 $\mathrm{mm}$ locking reconstruction plates. No significant difference in complication rates was reported between the two types of plates. Plate removal was necessary in $28 \%$, which is similar as the findings reported in the first mentioned study.

A total complication rate of $28 \%$ was reported, including loose screws (4.3\%), oral- (7.4\%) and extraoral dehiscences with fistula formation (11.7\%). 
Dehiscences were seen significantly more often in larger defects and with longer plates. Moreover, the rate of dehiscences was significantly higher in the midline. In conclusion, plate removal of 2.0 $\mathrm{mm}$ locking reconstruction plates is reported to be around $28 \%$, and the probability of a complication seems to increase with the size of the defect. Therefore, when assessing complication rates, it is important not only to take the type of osteosynthesis material into account, but also anatomic factors such as defect size or anatomic location of the fracture, which seem to have a significantly more important impact on the success rate of the osteosynthesis than the osteosynthesis material itself.

\subsubsection{Locking vs Non-Locking Plates/Screws}

2 systematic reviews with meta-analysis compared a $2.0 \mathrm{~mm}$ locking plate system vs a non-locking system. The double-threaded screws of locking 2.0 $\mathrm{mm}$ miniplates locking to the bone and the plate create a mini-internal fixator, which results in a more rigid construction with less distortion of the fracture or osteotomy, less screw loosening, and less interference with bone circulation due to the slight pressing of plates against the bone. In short, theoretical advantages of the locking miniplate system mainly include less precision required in plate adaptation because of the internal/external fixator, less alteration in osseous or occlusal relationship on screw tightening, greater stability across the fracture sites and less screw loosening [12]. One study found a cumulative RR of 0.79 , meaning the use of the locking plate in the fixation of mandibular fractures decreased the risk of the event (postoperative complication) by $21 \%$ [13]. However, despite the theoretical advantages of locking systems, neither of these systematic reviews found a significant difference in postoperative complication rates with the use of locking screw/plate systems when compared to the use of non-locking systems in the management of mandibular fractures.

\subsubsection{Bicortical Screws}

One study investigated the use of bicortical lag screws in 259 patients who had been treated by either BSSO or bimaxillary-osteotomy, with a total of 502 sagittal split osteotomies performed [14]. Removal of the osteosynthesis material was necessary in $5.6 \%$ of the cases. Bicortical screws had to be removed at $2.9 \%$ of the sites, and $2.3 \%$ of the removals were related to infection, including $1.2 \%$ of intra-oral fistula formation. In conclusion, the authors found that rigid fixation with 3 bicortical screws after $\mathrm{BSSO}$ is reliable with a low rate of postoperative removal of the osteosynthesis material. Other reported incidences indicate a lower rate of removal of bicortical screws than of miniplates [14]. Bicortical lag screw fixation was found to be at least as safe as miniplate fixation. Moreover, because of better fragment compression, bicortical lag screw fixation offers faster bone healing.

3.1.2. 3D (CAD/CAM) Materials

2 studies that compared 3D (CAD/CAM) plates and screws with standard titanium plates and screws were included. One retrospective study included 142 subjects who underwent microvascular mandibular reconstruction [3]. Perioperative complication rates were $35.9 \%$ in the titanium control Group vs $20.7 \%$ in the CAD/CAM custom plates Group. Hardware removal was necessary in $20.2 \%$ of the titanium plates vs $5.6 \%$ of the CAD/CAM custom plates. $\mathrm{CAD} / \mathrm{CAM}$ custom plates utilized for rigid fixation during microvascular mandibular reconstruction demonstrated fewer complications and statistically lower reoperation rates when compared with prebent/preformed titanium plates. The other study included was a systematic review and meta-analysis including a total of 661 patients in 17 studies that compared 3D miniplates with the standard twominiplate technique in mandibular fractures [2]. Mandibular fracture fixation with 3D miniplates was found to decrease the risk of postoperative complications by $52 \%$ compared with standard miniplates (OR 0.48). Infection and wound dehiscence were less common in the 3D miniplate Group (OR 0.58 ; OR 0.36 , respectively), but these findings were not significant. The cumulative analysis showed a statistically significant difference in the outcome of hardware failure, favoring 3D miniplates (OR 0.14, $p=0.004)$. The results of this meta-analysis showed that the use of 3D miniplate fixation had lower complication rates when compared with the use of standard miniplate fixation in the management of mandibular fractures. This result was statistically significant. Another interesting outcome measured in this systematic review was the operative time: 3D plate technique showed a significant time benefit $(p<0.00001)$. The authors concluded that the major advantage of the 3D miniplate technique is the simultaneous stabilization of the tension and compression zones, making the 3D plates a timesaving alternative to conventional miniplates.

3.1.3. Biodegradable Materials

The use of biodegradable materials for osteosynthesis has been an interesting evolution on the market. The theoretical advantage of their resorbable properties sounds promising, as it could resolve the problem of the need for a second surgery for removal of other non-resorbable materials. However, this feature does not tell much about the clinical usefulness of such materials, as it does not exclude other complications such as non-union, infection, hardware failure or removal. One systematic review and three RCT's were included in this report. The removal rates varied between $1.5 \%-16.4 \%$ in the titanium control Group and 3.6-26.4\% in the biodegradable test Group [5,15]. The risk of necessity for biodegradable plate and screws removal was two times higher compared to titanium plates and screws after long-term follow-up $>5 y$ (HR 2.0, $p=0.036$ ) [5]. Abscess formation was the main reason for plate/screw removal in both groups $[4,5]$. Regarding plate/screws removal after $>2$ and $>5$ years follow-up, the performance of the Inion CPS biodegradable system was inferior compared to the KLS Martin titanium system following the fixation of mandibular, Le Fort-I, and zygomatic fractures, and bilateral sagittal split osteotomies (BSSO) and/ or Le Fort-I osteotomies [4,5]. Given the higher rates of plate removal, there seems to be no place for the clinical usage of Inion CPS in treatment of these 
clinical usage of Inion CPS in treatment of these surgical indications. Another RCT included 200 Japanese adults with jaw deformities diagnosed as mandibular prognathism who underwent BSSO surgery [16]. The authors found complication rates of $8.2 \%$ in the biodegradable Group and 3.3\% in the titanium Group, including similar infection rates of $3.6 \%$ and $3.3 \%$, respectively. However, although a greater frequency of material-related complication was observed in the biodegradable fixation plate Group, no statistically significant differences were found in the incidence of material-related complications between the biodegradable and titanium groups. The authors suggest that the use of biodegradable plates should be recommended only for minimally loaded situations.

\subsection{Corrosion- and Particle Detection Rates}

In this second search, the focus was mainly set on the rates of corrosion and particle detection in soft tissues. An extended search was performed for both outcomes. Data were found reporting titanium particles detected in animal lymph nodes after osteosynthesis before $[17,18]$, and several articles mentioned the detection of titanium particles in locoregional lymph nodes, which are thought to be due to the corrosion of the titanium [15]. However, reports of titanium and titanium dioxide in tissues adjacent to hardware and in regional lymph nodes have shown that only clinically insignificant amounts of these materials accumulate [1]. The data analysis in this report focusses on the incidence of corrosion and pigmentation deposits from titanium fixation systems.

Thre studies that examined the outcomes of interest were included. Acero et al. carried out a prospective histological study on 37 commercially pure titanium miniplates, removed from 23 patients who had undergone surgery for maxillofacial traumatic injuries or deformities [18]. Hole-like images were found in $35.1 \%$ of the plates studied. The authors suggest that such anomalies may be corrosion effects in a biomaterial, with titanium-particles released to the surrounding tissues. Dark pigmented deposits were found in $80 \%$ of the specimens of soft tissue surrounding the analyzed plates. Defects on the surface of the biomaterial and pigmented deposits in soft tissues surrounding the plates suggest a higher development of corrosion in titanium than previously reported. In a more recent study, 60 pure titanium plates retrieved from 44 patients and 60 soft tissue specimens taken from adjacent locations were examined [19]. Pigmented deposits were detected in $68 \%$ of the soft tissue specimens. These pigmented deposits were initially also attributed to the presence of titanium particles, as in most similar studies. However, elemental analysis of pigmented deposits did not confirm this assumption. The authors concluded that the incidence of 'titanium deposits' might have been overestimated in the past. Moreover, this study did not report any signs of corrosion of the metal plates. These findings correspond to the results of the third study included, where no evidence of macroscopic or microscopic titanium corrosion or deterioration in the tissues was found [20]. There was no evidence to support the view that titanium miniplates should be removed routinely due to corrosion up to a period of 13 years. Overall, these findings suggest that corrosion and particle deposition in surrounding soft tissues are clinically irrelevant and should not be considered as a reason for the removal of titanium osteosynthesis material on the long-term.

\section{CONCLUSION}

This review provides an overview of the complication rates related to different types of osteosynthesis materials and systems used in maxillofacial surgery, including rejection-, removal-, corrosion- and particle detection rates in surrounding tissues.

Reported incidences of removal of titanium fixation systems in maxillofacial surgery ranged from 5,6\% to $28 \%$, depending on the type of titanium fixation system and the surgical indication. When comparing titanium- to 3D (CAD/CAM) materials, reported hardware removal rates are up to four times lower for 3D manufactured materials. Moreover, the use of the 3D miniplate fixation has significantly lower complication rates in the management of mandibular fractures. Therefore, CAD/CAM fixation systems are recommended in the management of mandibular fractures. Biodegradable materials, however, are not recommended for clinical usage in the treatment of traumatic fractures or osteotomies in the maxillofacial area, given the higher rates of plate removal after $>5 y$ follow-up.

Finally, corrosion and particle deposition in the surrounding soft tissues seem to be clinically irrelevant and should not be considered as a reason for removal of the titanium osteosynthesis material on the long-term.

\section{CONFLICT OF INTEREST}

The authors declare no conflict of interest.

\section{ACKNOWLEDGMENTS}

None.

\section{AUTHOR CONTRIBUTIONS}

SEB: data acquisition, analysis and interpretation of the results, author of the article. CP: substantial contribution to the conception and design of the study, revision of the manuscript. 


\section{Appendix I: Pubmed search strategy}

\section{Search A:}

( "Oral Surgical Procedures"[Mesh] OR "Orthognathic Surgical Procedures"[Mesh] OR "Surgery, Oral"[Mesh] OR "Maxillofacial Prosthesis Implantation"[Mesh] OR "Orthognathic Surgery"[Mesh] OR "Facial Bones/ surgery"[MAJR] OR "Mandibular Reconstruction"[Mesh]) AND ("Fracture fixation, Internal"[MESH] OR"Fracture fixation" ${ }^{*}[T I A B]$ OR "titanium plate ${ }^{* \prime}[T I A B]$ OR "Reconstruction plate ${ }^{*}$ " $[T I A B]$ OR "Bone plates"[MESH] OR "Bone plate*"[TIAB] OR "Bone screws" [MESH] OR "Bone screw*"[TIAB] OR "Plate fixation*"[TIAB] OR "Fixation plate ${ }^{* \prime[}[T I A B]$ OR "osteosynthesis plate *"[TIAB] OR "Bone Plates*/adverse effects"[MAJR] OR "osteosynthesis material*"[TIAB]) AND ("rejection*"[TIAB] OR"EquipmentFailure"[MeSHTerms] OR"Infections"[Mesh]OR "Infection*"[TIAB] OR"screw loosening"[TIAB] OR"loosened screws"[TIAB] OR"plate exposure"[TIAB] $O R$ "plate extrusion" [TIAB] OR "wound dehiscence"[TIAB] OR "Device removal" [MESH] OR "Device removal*"[TIAB] OR "plate removal*"[TIAB] OR "Hardware removal"[TIAB]) AND (("randomized controlled trial"[PT] OR "controlled clinical trial"[PT] OR "clinical trial" $[\mathrm{PT}] \mathrm{OR}$ "comparative study" [PT] OR "Cross-Over Studies"[Mesh] OR "Intervention Studies"[Mesh] OR random*[TIAB] OR controll*[TIAB] OR "intervention study"[TIAB] OR "experimental study"[TIAB] OR "comparative study"[TIAB] OR trial[TIAB] OR evaluat*[TIAB] OR "Before and after"[TIAB] OR "interrupted time series"[TIAB]) NOT ("animals"[MH] NOT (animals[MH] AND "humans"[MH])))

\section{Search B:}

( "Oral Surgical Procedures"[Mesh] OR "maxillofacial"[TIAB] OR "Orthognathic Surgical Procedures"[Mesh] OR "Surgery, Oral"[Mesh] OR "Maxillofacial Prosthesis Implantation"[Mesh] OR "Orthognathic Surgery"[Mesh] OR "Facial Bones/surgery"[MAJR] OR "Mandibular Reconstruction"[Mesh]) AND ("Fracture fixation, Internal" [MESH] OR "Fracture fixation*"[TIAB] OR "titanium plate*"[TIAB] OR "Reconstruction plate*"[TIAB] OR "Bone plates"[MESH] OR "Bone plate*"[TIAB] OR "Bone screws"[MESH] OR "Bone screw*"[TIAB] OR "Plate fixation*"[TIAB] OR "Fixation plate*"[TIAB ] OR "osteosynthesis plate*"[TIAB] OR "Bone Plates*/adverse effects"[MAJR] OR "osteosynthesis material*"[TIAB]) AND ("Corrosion"[MESH] OR "Corrosion"[TIAB] OR ("Biocompatible Materials/chemistry"[MAJR]) OR "Titanium/chemistry"[MAJR] OR "particle detection"[TIAB] OR "pigment deposition"[TIAB]) AND (("randomized controlled trial"[PT] OR "controlled clinical trial"[PT] OR "clinical trial"[PT] OR "comparative study"[PT] OR "Cross-Over Studies"[Mesh] OR "Intervention Studies"[Mesh] OR random*[TIAB] OR controll*[TIAB] OR "intervention study"[TIAB] OR "experimental study"[TIAB] OR "comparative study"[TIAB] OR trial[TIAB] OR evaluat*[TIAB] OR "Before and after"[TIAB] OR "interrupted time series"[TIAB]) NOT ("animals"[MH] NOT (animals[MH] AND "humans"[MH]))

\section{REFERENCES}

1. Haug RH. Retention of asymptomatic bone plates used for orthognathic surgery and facial fractures. J Oral Maxillofac Surg. 1996;54(5):611-6177. doi: 10.1016/S0278-2391(96)90644-8. CrossRef Full text links PubMed Google Scholar Scopus

2. Wusiman $P$, Yarbag A, Wurouzi G, et al. Three dimensional versus standard miniplate fixation in management of mandibular fractures: A systematic review and meta-analysis. J CranioMaxillofacial Surg. 2016;44(10):1646-1654. doi: 10.1016/j. jcms.2016.07.027.

CrossRef PubMed Google Scholar Scopus

3. McCann AC, Shnayder Y, Przylecki WH, et al. Comparison of modern rigid fixation plating outcomes for segmental mandibular microvascular reconstruction. Laryngoscope. 2019;129(5):1081-1086. doi: 10.1002/lary.27406. CrossRef PubMed Google Scholar Scopus

4. Van Bakelen NB, Buijs GJ, Jansma J, et al. Comparison of biodegradable and titanium fixation systems in maxillofacial surgery: A two-year multi-center randomized controlled trial. J Dent Res. 2013;92(12):1100-1105. doi: $10.1177 / 0022034513508953$.

CrossRef Full tekst links PubMed Google Scholar Scopus 5. Gareb B, Van Bakelen NB, Buijs GJ, et al. Comparison of the long-term clinical performance of a biodegradable and a titanium fixation system in maxillofacial surgery: a multicenter randomized controlled trial. PLoS One [Internet]. 2017;12(5):1-12. doi: 10.1371/journal.pone.0177152

CrossRef Free PMC Article PubMed Google Scholar Scopus 6. Zhang ZL, Wang S, Sun CF, et al. Miniplates versus reconstruction plates in vascularized osteocutaneous flap reconstruction of the mandible. J Craniofac Surg. 2019;30(2):E119-E125. doi: 10.1097/SCS.0000000000005020. CrossRef PubMed Google Scholar Scopus

7. Ellis E. A study of 2 bone plating methods for fractures of the mandibular symphysis/body. J Oral Maxillofac Surg. 2011;69(7):1978-1987. doi: 10.1016/j.joms.2011.01.032. CrossRef PubMed Google Scholar Scopus

8. Kim MY, Kim CH, Han SJ, et al. A comparison of three treatment methods for fractures of the mandibular angle. Int J Oral
Maxillofac Surg [Internet]. 2016;45(7):878-883.

doi: 10.1016/j.ijom.2016.02.013

CrossRef PubMed Google Scholar Scopus

9. Ellis E. A prospective study of 3 treatment methods for

isolated fractures of the mandibular angle. J Oral Maxillofac Surg [Internet]. 2010;68(11):2743-2754.

doi: $10.1016 /$ j.joms.2010.05.080

CrossRef PubMed Google Scholar Scopus

10. Wood CB, Shinn JR, Amin SN, et al. Risk of plate removal in free flap reconstruction of the mandible. Oral Oncol. 2018;83(June):91-95. doi: 10.1016/j.oraloncology.2018.06.008. CrossRef PubMed Google Scholar Scopus

11. Kämmerer PW, Klein MO, Moergel M, et al. Local and systemic risk factors influencing the long-term success of angular stable alloplastic reconstruction plates of the mandible.

J Cranio-Maxillofacial Surg [Internet]. 2014;42(5):e271-e276. doi: 10.1016/j.jcms.2013.10.004. [CrossRef] [PubMed] Google Scholar Scopus

12. Zhan S, Jiang Y, Cheng Z, et al. A meta-analysis comparing the $2.0-\mathrm{mm}$ locking plate system with the $2.0-\mathrm{mm}$ nonlocking plate system in treatment of mandible fractures. J Craniofac Surg. 2014;25(6):2094-2097. doi: 10.1097/SCS.0000000000001018. CrossRef PubMed Google Scholar Scopus

13. Chrcanovic BR. Locking versus non-locking plate fixation in the management of mandibular fractures: a meta-analysis. Int $J$ Oral Maxillofac Surg [Internet]. 2014;43(10):1243-1250.

doi: 10.1016/j.ijom.2014.07.014.

CrossRef PubMed Google Scholar Scopus

14. Verweij JP, Houppermans PNWJ, Mensink G, et al. Removal of bicortical screws and other osteosynthesis material that caused symptoms after bilateral sagittal split osteotomy: a retrospective study of 251 patients, and review of published papers. Br J Oral Maxillofac Surg [Internet]. 2014;52(8):756-760. doi: 10.1016/j. bjoms.2014.05.017.

CrossRef PubMed Google Scholar $\underline{\text { Scopus }}$ 
15. Agnihotry A, Fedorowicz Z, Nasser M, et al. Resorbable versus titanium plates for orthognathic surgery. Cochrane Database Syst Rev. 2017;2017(10)

doi: 10.1002/14651858.CD006204.pub3

CrossRef Free PMC Article PubMed Google Scholar Scopus

16. Yoshioka I, Igawa K, Nagata J, et al. Comparison of materialrelated complications after bilateral sagittal split mandibular setback surgery: biodegradable versus titanium miniplates. J Oral Maxillofac Surg [Internet]. 2012;70(4):919-924. doi: 10.1016/j. joms.2011.02.136.

CrossRef PubMed Google Scholar Scopus

17. Fage SW, Muris J, Jakobsen SS, et al. Titanium: a review on exposure, release, penetration, allergy, epidemiology, and clinica reactivity. Contact Dermatitis. 2016;74(6):323-345.

doi: $10.1111 /$ cod.12565.

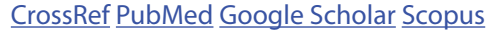

18. Acero J, Calderon J, Salmeron Jl, et al. The behaviour of titanium as a biomaterial: microscopy study of plates and surrounding tissues in facial osteosynthesis. J CranioMaxillo-Facial Surg. 1999;27(2):117-123. doi: 10.1016/s10105182(99)80025-0.

CrossRef PubMed Google Scholar Scopus

19. Theologie-Lygidakis N, latrou I, Eliades G, et al. A retrieval study on morphological and chemical changes of titanium osteosynthesis plates and adjacent tissues. J Cranio-Maxillofacial Surg. 2007;35(3):168-176. doi: 10.1016/j.jcms.2007.01.004.

CrossRef PubMed Google Scholar Scopus

20. Langford RJ, Frame JW. Surface analysis of titanium maxillofacial plates and screws retrieved from patients. Int J Oral Maxillofac Surg. 2002;31(5):511-518. doi: 10.1054/ijom.2002.0283. [CrossRef] [PubMed] Google Scholar Scopus

\section{Samy EI BACHAOUI}

MD, Master's Student in Medicine Department of Oral and Maxillofacial Surgery Faculty of Medicine University of Leuven Leuven, Belgium

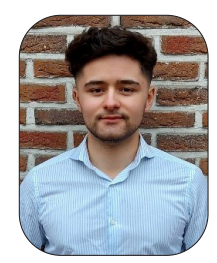

Samy El Bachaoui is a Master's student in Medicine at the University of Leuven (KU Leuven) with a special interest in Oral- and Maxillofacial surgery. He obtained his bachelor's degree in Medicine in 2018 (cum laude), and is expected to obtain his master's degree in Medicine in June 2021. He has gained experience with internships in the department of Oral- and Maxillofacial Surgery at the Geneva University Hospitals and the University Hospitals Leuven under the supervision of Professor Dr. Constantinus Politis.

\section{Ouestions}

\section{What fixation system has the lowest complication rates and is therefore recommended in the management of mandibular fractures?}

口a. Double titanium miniplate system;

ab. Biodegradable materials;

c. 3D (CAD/CAM) fixation systems;

ad. Locking plates/screws.

\section{The risk of postoperative complications is:}

$\square$ a. Decreased by approximately 50\% with 3D miniplates compared to titanium plates in the management of mandibular fractures;

b. Three times higher in terms of hardware removal with biodegradable materials compared to titanium plates;

ac. Significantly lower for double miniplates compared to single miniplates;

$\square$ d. Not significantly different for double titanium miniplates compared to a large reconstruction plate in the management of mandibular fractures.

\section{Regarding plate/screws removal after $>5$ years follow-up following fixation of traumatic fractures and osteotomies in the maxillofacial area, the performance of the biodegradable system was assessed as:}

$\square$ a. Superior compared to the titanium system, therefore the clinical usage of a biodegradable fixation system in the treatment of these surgical indications is strongly recommended;

bb. Superior compared to the titanium system, therefore the use of biodegradable plates should be recommended for maximally loaded situations;

ac. Equal compared to the titanium system, therefore the use of biodegradable plates could be a clinically useful alternative in the treatment of these surgical indications;

$\square$ d. Inferior compared to the titanium system, therefore there seems to be no place for the clinical usage of biodegradable systems in the treatment of these surgical indications.

\section{Which of the following statements is true regarding corrosion and/or particle deposition in surrounding soft tissues?}

$\square$ a. Corrosion rates of titanium miniplates are clinically relevant and should be considered as a reason for the removal of titanium osteosynthesis material on the long-term;

b. Corrosion rates of titanium miniplates are clinically irrelevant and should not be considered as a reason for the removal of titanium osteosynthesis material on the long-term;

ac. Pigment deposition rates in surrounding soft tissues of titanium plates of up to $90 \%$ have been reported and should therefore be considered as a reason for the removal of titanium osteosynthesis material on the long-term;

d. Reports of titanium deposits show no evidence of particle accumulation in tissues adjacent to titanium hardware nor in regional lymph nodes. 